\title{
A Step Toward Moving Forward in Autonomic Nervous System Research
}

Can J Neurol Sci. 2013; 40: 767

Autonomic nervous system (ANS) is responsible for regulation and integration of the functions of internal organs. Broadly, ANS disorders can be divided into structural (characterized by minimal symptoms with demonstrable pathologic abnormalities that directly affect autonomic function) or functional (primarily defined by symptomology with no consistently demonstrable pathology). One of the most common presentations of dysautonomia is orthostatic intolerance characterized by symptoms upon standing and relieved by recumbency ranging from uneasiness, discomfort, lightheadedness, palpitations, headache and anxious feeling. There is no wonder that these patients are often incorrectly labeled as having panic disorder or chronic anxiety and are referred to psychiatrists. ${ }^{1}$

Over the past two decades, improved testing has allowed us to recognize not only new syndromes characterized by orthostatic intolerance (such as postural orthostatic tachycardia syndrome), but also to recognize the high percentage of ANS involvement in neurological diseases such as multiple sclerosis. ${ }^{2}$

If we know that symptoms attributable to ANS involvement are frequent in both general population (mostly functional ANS disorder) as well as population suffering from various neurological diseases (mostly structural ANS disorder), it is surprising that testing of the ANS is still not widely used and available. There are several reasons for this: 1) more tests of autonomic function exist than for any other neurological system; 2) autonomic function tests are indirect, relatively imprecise, and fraught with difficulties; 3) numerous technical and physiologic variables must be controlled for; autonomic regulation is slow, intricate, and complex; and the visceral physiological systems being regulated are themselves intricate and complex. ${ }^{3}$ Moreover, standardization of test conditions is crucial in order to make them comparable. This is especially true for the assessment of cardiovascular reflexes and sudomotor function.

Despite these obstacles, these tests can give us critical insight into ANS functions. Clinical goals in the evaluation of the autonomic function include but are not limited to: evaluation of the severity and distribution of autonomic dysfunction, diagnosis of autonomic neuropathy, diagnosis and evaluation of orthostatic intolerance, monitoring the course of dysautonomia, monitoring response to treatment, and as an instrument in research studies. ${ }^{4}$

In order to improve the reproducibility and clinical usefulness of these tests, it is essential that every laboratory use standardized data based on measurements obtained from a single laboratory and sample population. In this issue of the Canadian Journal of Neurological Sciences, Ives and colleagues present data on quantitative sudomotor axon reflex testing (QSART), heart rate response to deep breathing, and Valsalva maneuver obtained from 121 healthy participants from Southwestern Ontario. ${ }^{5}$

Besides being the first study to report sudomotor data and establish a normative database for QSART using a gel-based vehicle for delivery of ACh in conjunction with the Q-Sweat apparatus, the results of this study have confirmed several previously published findings: 1) a significant effect of gender on QSART results, with greater sweat volumes for men than for women; 2) a significant effect of age on QSART results, with lower mean sweat volumes with age at the distal leg and foot sites; 3) a reduction in heart rate variation with age; and 4) a significant effect of age on Valsalva ratio results. Furthermore, this study showed that the total sweat volume is a better measure of postganglionic sudomotor axon integrity than the time to sweating onset following application of the stimulus.

This study has provided clinicians and researchers from Canada with an important tool for evaluation of ANS dysfunction and is a step forward in ANS research. This is of particular importance as ANS dysfunction must be actively sought for by the neurologist and appropriately treated, as it greatly affects the patients' quality of life as well as their social functioning. Further work is still needed to establish normative values for the head-up tilt test as a part of autonomic reflex screen in order to better define functional disorders of the ANS.

\author{
Mario Habek \\ Laboratory for Autonomic Nervous System Testing \\ Department of Neurology, University Hospital Center Zagreb \\ University of Zagreb, School of Medicine, Zagreb, Croatia \\ Email:mhabek@mef.hr
}

\section{REFERENCES}

1. Khurana RK. Experimental induction of panic-like symptoms in patients with postural tachycardia syndrome. Clin Auton Res. 2006;16(6):371-7.

2. Adamec I, Lovrić M, Žaper D, et al. Postural orthostatic tachycardia syndrome associated with multiple sclerosis. Auton Neurosci. 2013;173(1-2):65-8.

3. Ravits JM. AAEM minimonograph \#48: autonomic nervous system testing. Muscle Nerve. 1997;20(8):919-37.

4. Low PA, Tomalia VA, Park KJ. Autonomic function tests: some clinical applications. J Clin Neurol. 2013;9(1):1-8.

5. Ives CT, Berger MJ, Kimpinski K. The autonomic reflex screen in healthy participants from Southwestern Ontario. Can J Neurol Sci. 2013;40(6):848-53. 